\title{
Development of a Pulsed Combustion Actuator For High-Speed Flow Control
}

\author{
Andrew. D. Cutler * \\ The George Washington University, Hampton, VA, 23666 \\ B. Terry Beck ${ }^{\dagger}$ \\ Kansas State University, Manhattan, KS, 66506 \\ Jennifer A. Wilkes ${ }^{\ddagger}$ \\ The College of William and Mary, Williamsburg, VA, 23187 \\ and \\ J. Philip Drummond ${ }^{\S}$, David W. Alderfer ${ }^{* *}$, and Paul M. Danehy ${ }^{\dagger+}$ \\ NASA Langley Research Center, Hampton, VA, 23681
}

\begin{abstract}
This paper describes the flow within a prototype actuator, energized by pulsed combustion or detonations, that provides a pulsed jet suitable for flow control in high-speed applications. A high-speed valve, capable of delivering a pulsed stream of reactants-a mixture of $\mathrm{H}_{2}$ and air - at rates of up to 1500 pulses per second, has been constructed. The reactants burn in a resonant chamber, and the products exit the device as a pulsed jet. High frequency pressure transducers have been used to monitor the pressure fluctuations in the device at various reactant injection frequencies, including both resonant and off-resonant conditions. The combustion chamber has been constructed with windows, and the flow inside it has been visualized using Planar Laser-Induced Fluorescence (PLIF). The pulsed jet at the exit of the device has been observed using schlieren.
\end{abstract}

\section{Introduction}

\section{A. Background \\ B OUNDARY layer control can be broadly classified into passive and active control ${ }^{1}$. Common applications Binclude the control of boundary layer separation in external flows to maintain the effectiveness of flaps and other control surfaces, and internal flow boundary layer control associated with the operation of jet engine inlet and diffuser flows. Passive control includes the use of common devices such as vortex generator tabs ${ }^{2}$ to delay the onset of boundary layer separation. Active control includes the many leading and trailing edge devices used in conjunction with flow control over wing surfaces ${ }^{3}$. \\ Actuators of various types play an important role in active boundary layer control. A variety of different actuator devices have previously been investigated as flow control devices for both internal and external boundary layer applications. The operating principles for such devices are typically based on either mechanical deflection, mass injection, or the use of synthetic jets ${ }^{4}$. Mechanical actuator devices include conventional flap controls, as well as more recent MEMS flap devices. Mass injection actuators are characterized by a net mass flow through the

\footnotetext{
* Associate Professor, MAE Dep't, 303 Butler Farm Rd. Suite 101A, Associate Fellow AIAA

${ }^{\dagger}$ Professor, Dep't of Mechanical \& Nuclear Eng., 302 Rathbone Hall.

${ }^{\ddagger}$ Graduate Student, Dep’t of Physics, P.O. Box 8795, Student Member AIAA.

$\S$ Senior Research Scientist, Hypersonic Air Breathing Propulsion Branch, MS 168. Associate Fellow AIAA

${ }^{* *}$ Electronics Engineer, Advanced Sensing and Optical Measurement Branch, MS 236.

${ }^{+\dagger}$ Optical Physicist, Advanced Sensing and Optical Measurement Branch, MS 236. Associate Fellow AIAA
}

Copyright (C 2005 by the American Institute of Aeronautics and Astronautics Inc. The U.S. Government has a royalty-free license to exercise all rights under the copyright claimed herein for Governmental purposes. All other rights are reserved by the copyright owner. 
actuator, and include combustion-driven jet actuators ${ }^{5}$. Synthetic jets are diaphragm devices that produce a pulsating jet for control of the boundary layer with no net mass injection ${ }^{6}$.

In high-speed applications, pulsed or synthetic jet actuators require both high impulse and high frequency. It is claimed $^{7}$ that dimensionless pulsation frequencies, defined as $\{$ frequency $\times$ streamwise length of separation $\} /\{$ free stream velocity\}, of $1-10$ are required for boundary-layer separation control (i.e., mitigation or removal of separation). This leads to the requirement for kilohertz level pulsation rates.

\section{B. Pulsed Combustion Actuator Concept}

Combustion jet actuators offer the promise of increased impulse in supersonic flows due to their utilization of the highly energetic combustion process. One such device, based on a deflagration (or subsonic wave) combustion process, is sparked to initiate combustion, and operates at a time scale between pulses very much greater than the time scales of wave propagation within the device ${ }^{8}$.

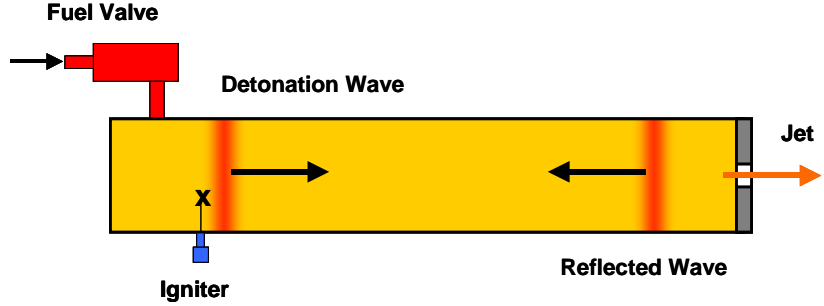

Figure 1. Resonant Detonation Actuator

A new type of combustion-driven pulsed-jet actuator has been proposed ${ }^{9}$ that would be capable of providing both high impulse and high frequency. The actuator may be similar to a small pulsejet engine ${ }^{10}$ or pulsed detonation engine (PDE) ${ }^{11}$ in that it operates in a resonant mode, taking advantage of wave propagation and reflection within the device to initiate or augment the combustion. Ideally, such a device would be fueled passively, i.e., fuel and air would be periodically drawn into the device as a consequence of the pressure variations associated with the wave motions, and might use aerodynamic or reed oneway valves. Since operation of a pulsejet or PDE has not been demonstrated at the high frequencies of interest to us (typical frequencies demonstrated by a PDE are $\sim 10-100 \mathrm{~Hz}$ ), the present conservative approach has been to actively fuel the device using a high-speed valve and to defer to the future development of passive fueling methods. The fuel/oxidant being considered is $\mathrm{H}_{2}$ /air, both because of the relative ease in ignition and detonation of these gases, and because of their ease of handling.

A simplified schematic of a combustion actuator device currently under investigation is shown in Fig. 1. It consists of an inlet valve to periodically inject a charge of $\mathrm{H}_{2}$ /air mixture, an igniter to initiate combustion, a tube in which the combustion takes place, and some type of exit through which the products are discharged into the atmosphere (or flow field to be controlled). Note that this study is limited to the development of the actuator and not to its application, so discharge is into stagnant laboratory air. In the figure, the exit consists of a plate with a nozzle.

Multiple resonances can occur, as illustrated in Fig. 2. This figure shows the relative amplitude of sinusoidal pressure fluctuations as a function of position along the tube, where negative amplitude indicates a $180^{\circ}$ phase delay. Also shown are the relative locations of three pressure gauges used in the experiment ( $\mathrm{p} 2, \mathrm{p} 3$, and $\mathrm{p} 4)$. In this figure, the

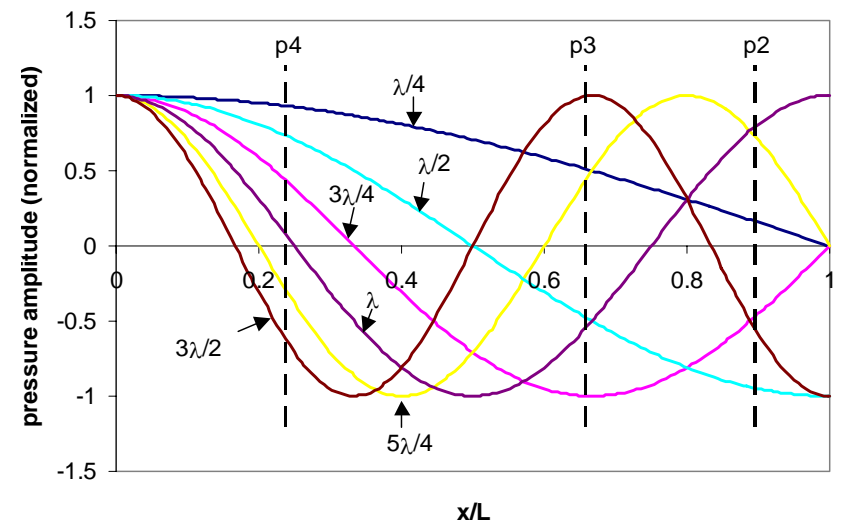

Figure 2. Amplitude of sinusoidal pressure fluctuations for various resonances as a function of distance along the tube. Experimental pressure taps are located at p2, p3, and $\mathbf{p 4}$. left hand side is the reactants injection side $(x=0)$, and the right is the discharge side $(x=L)$.

With the nozzle exit in place, as illustrated in Fig. 1 , the $\lambda / 2, \lambda$, 3 $\lambda / 2$, etc. modes are possible. The fundamental $(\lambda / 2)$ resonance occurs where a compression or shock wave emitted from the left side propagates to the right, is reflected as a compression or shock wave from the nozzle exit, and returns to the left side coincident with the next compression wave being emitted from the left. The identifier " $\lambda / 2$ " implies that, for sinusoidal variation of pressure in time at the left hand side, the pressure field in the tube at any given time approximately forms half a cosine wave, with a maxima/minima at the left- and right-hand sides and a zero point (node) at the center. The $2^{\text {nd }}$ harmonic $(\lambda)$ resonance occurs where the delay between the wave emission and return to the left-hand side coincides with two 


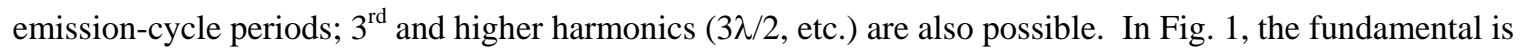
illustrated: fuel is ignited (ideally as a detonation), sending a compression or shock wave to the right. This wave is reflected as a compression or shock wave that propagates to the left, igniting (or accelerating the combustion) of a new fuel charge injected at the left side.

If the nozzle exit is removed, leaving the tube end open, the $\lambda / 4,3 \lambda / 4,5 \lambda / 4$, etc. modes are possible. This is the pulsejet or PDE mode. In this case a compression or shock wave incident upon the exit is reflected back into the tube as an expansion wave, and an expansion is reflected as a compression or shock. The fundamental $(\lambda / 4)$ resonance occurs where a compression emitted from the left is reflected from the right as an expansion, is reflected from the left as an expansion, is reflected from the right as a compression, and is incident upon the left side coincident with a new compression being emitted. The $2^{\text {nd }}(3 \lambda / 4), 3^{\text {rd }}(5 \lambda / 4)$, etc. harmonics are also possible.

Resonant frequencies may be computed from the following equation:

$$
f=\frac{a}{L}\left\{\frac{\text { Modeidentifier }}{\lambda}\right\} \quad \text { Equation } 1
$$

In this equation, $a$ is the speed of sound (or wave speed), and Modeidentifier is $\lambda / 4, \lambda / 2,3 \lambda / 4$, etc.

The ideal model described in which acoustic resonances occur is not likely to accurately describe a real actuator. If the actuator is to be effective in producing a large amplitude pulsating jet, pressure fluctuation amplitudes will be large and "linear," acoustic assumptions will not hold. Shock waves and detonation waves may occur.

Finally, an important feature of this concept, which distinguishes it from many pulse detonation engine or pulsejet concepts, is that the reactants are not ignited by an ignitor at each injection cycle, but are ignited simply by contact with the products of reaction from the previous cycle and by passage of a reflected wave. An ignitor (a spark plug) initiates reaction (at the first cycle) and thereafter plays no role in the process. Products are not displaced from the device by a non-reacting gas prior to injection of the next charge of reactants. This feature simplifies the fuel and air supply handling issues, and is suitable for high frequency applications.

\section{Experimental Method}

\section{A Actuator Model}

The current investigators have studied a number of different actuator configurations, with different inlet fuel-air port locations, as well as different combustion chamber lengths and exit geometries. This paper discusses only two configurations, which differ only in the exit geometry. These particular configurations have been studied in much greater detail than others.

A rendered cut-away view of the actuator configuration considered in this paper is shown in Fig. 3(a), and a photograph of the actual device is shown in Fig. 3(b). A motor drives a high-speed valve assembly (discussed below). A pulsed jet of $\mathrm{H}_{2}$-air mixture enters the windowed combustor from the left at $x=1.15$ inches (from the bottom). The actuator has a 1.52 in. wide by 1.19 in. deep rectangular combustor section near the bottom. Between $x=6$ and $x=8$ inches from the bottom, it transitions to a circular cross section of $1.52 \mathrm{in}$. diameter. The rectangular and circular cross-sectional areas are the same. (This non-constant cross section shape came about because of the desire to reuse tubular section components of a previous configuration, along with the requirement that a rectangular section be used for the windowed portion of the combustor.) The tube may be closed at $x=L=16.85$ inches by a short nozzle section with a 0.625 in. throat diameter, or the nozzle can be removed so that the tube discharges directly to the atmosphere. An automobile spark plug is located directly opposite the reactants injection hole to provide an initial ignition source. For safety reasons, the spark plug is actually fired continuously at $120 \mathrm{~Hz}$, independently of the of the injection process that occurs at frequencies from $100 \mathrm{~Hz}$ to $1500 \mathrm{~Hz}$. The spark appears to play little role in the combustion after initial ignition.

The combustor section has windows both front and back that extend from $x=0$ to $x=6$ inches, and the full width of the combustor (1.52 inches), to allow visualization of the flow in the device. Additionally, a window is provided at the bottom, beneath a narrow slot, to allow a laser light sheet to enter the model along the symmetry plane (the plane of the cut in Fig. 3(a)). Windows were made from 1-inch thick ultraviolet-grade fused silica to allow transmission of both the laser light and the resulting fluorescence from the $\mathrm{OH}$ radical, both of which are in the ultraviolet.

A rendered view of the valve assembly, with the valve body cut away in a plane through the $\mathrm{H}_{2}$ and air supply passages, is shown in Fig. 4(a). A photograph of the actual valve body (without the motor) is shown in Fig. 4(b), and a detail of the rotating shaft is shown in Fig. 4(c). The valve consists of a rotating shaft, which is supported by 
high-speed bearings, with a hole (for $\mathrm{H}_{2}$ ) and a slot (for air). The shaft rotates in a housing containing passages for the $\mathrm{H}_{2}$ and air and a hole and slot slightly larger than the corresponding hole and slot in the shaft. When the holes and slots in the shaft and housing align — which occurs twice per shaft revolution- $\mathrm{H}_{2}$ and air pass through the valve and are combined in the small nozzle, prior to entering the combustor. The holes and slots are designed to provide a near stoichiometric mixture of hydrogen and air when the inlet supply pressures are equal.

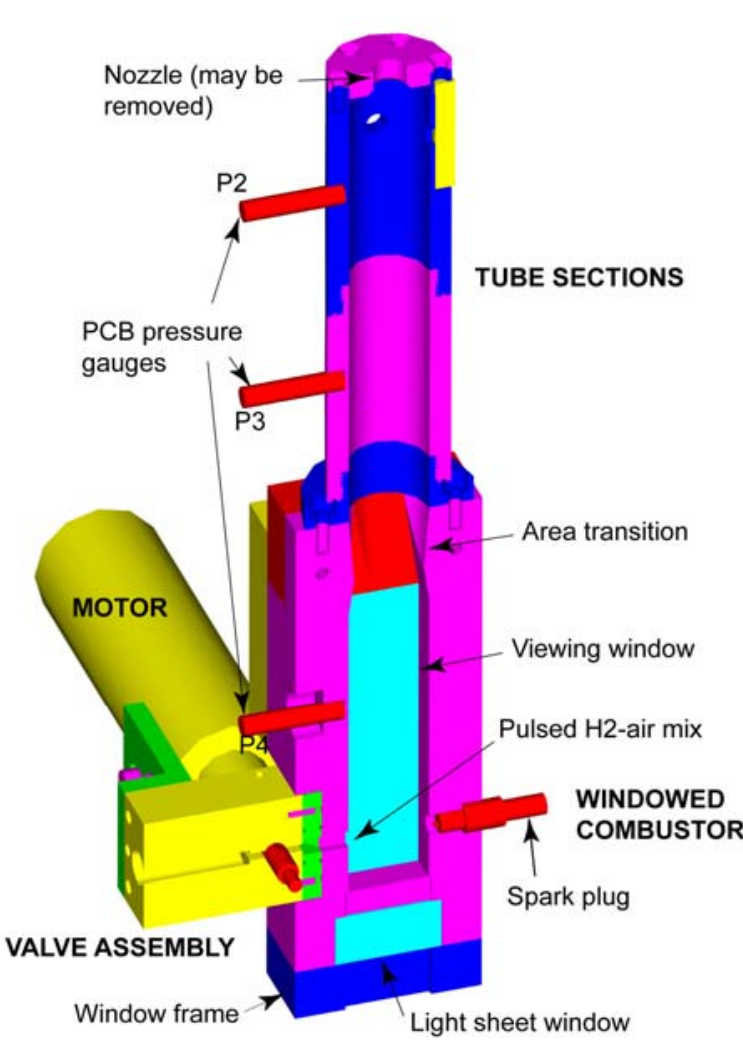

(a)

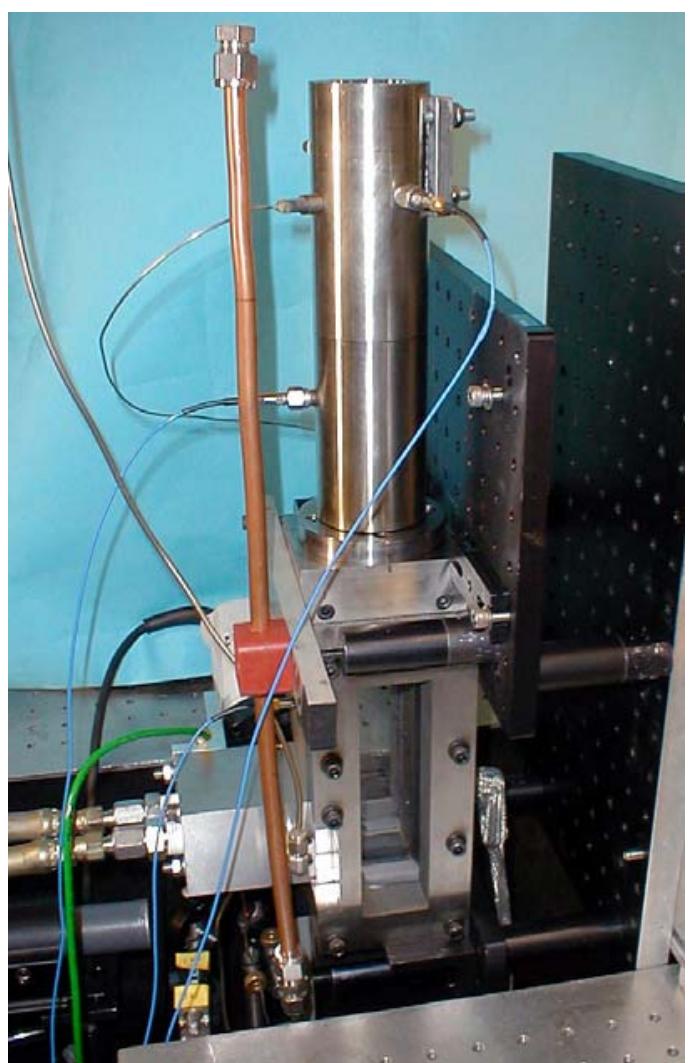

(b)

Figure 3. (a) Partially cut away view of actuator. (b) Photograph of mounted actuator assembly.

A high-speed (up to 45,000 rpm) "universal” AC/DC motor turns the rotating shaft. This motor was manufactured to drive a range of interchangeable "homogenizer" tools; the valve is interchangeable with these tools, allowing quick disconnect of the motor. The motor speed is controlled by a DC motor controller, which can be set manually or by an external 0-10 V DC control voltage. This controller, however, cannot effectively maintain a constant speed. An electronic "proportional-integral-differential," or PID, speed controller is available that receives the TTL pulses from the motor as feedback and outputs a control voltage to the motor controller: it is capable of maintaining speed within $1 \%$ of setpoint. Pressure taps are provided in the valve for monitoring both inlet hydrogen and inlet air pressures.

The valve and actuator were fabricated from various grades of stainless steel. They were not actively cooled. However, they were designed to be massive, with thick walls and windows, and thus acceptable run times of $\sim 20$ seconds were possible before the temperatures became high enough to be of concern. With experience, this was found to be adequate time to both establish the desired $\mathrm{H}_{2}$ and air flow rates and to acquire sufficient data. After a run, blowing air through the valve and combustor cooled the apparatus.

\section{Miscellaneous Instrumentation}

Pressurized air and gaseous hydrogen fuel were supplied separately to the actuator through the mixing valve inlet ports. Flow of $\mathrm{H}_{2}$ was measured using a 0-1000 standard liter per minute (SLPM) Hastings mass flow meter and flow of air was measured using a 0-2500 SLPM Hastings mass flow meter. 
Valve shaft position was measured by means of a helium-neon laser aimed at the rotating shaft, a retroreflective spot on the shaft, and a photodiode to detect reflected laser light. A custom-fabricated electronic package was used to convert the photodiode signal to a TTL pulse.
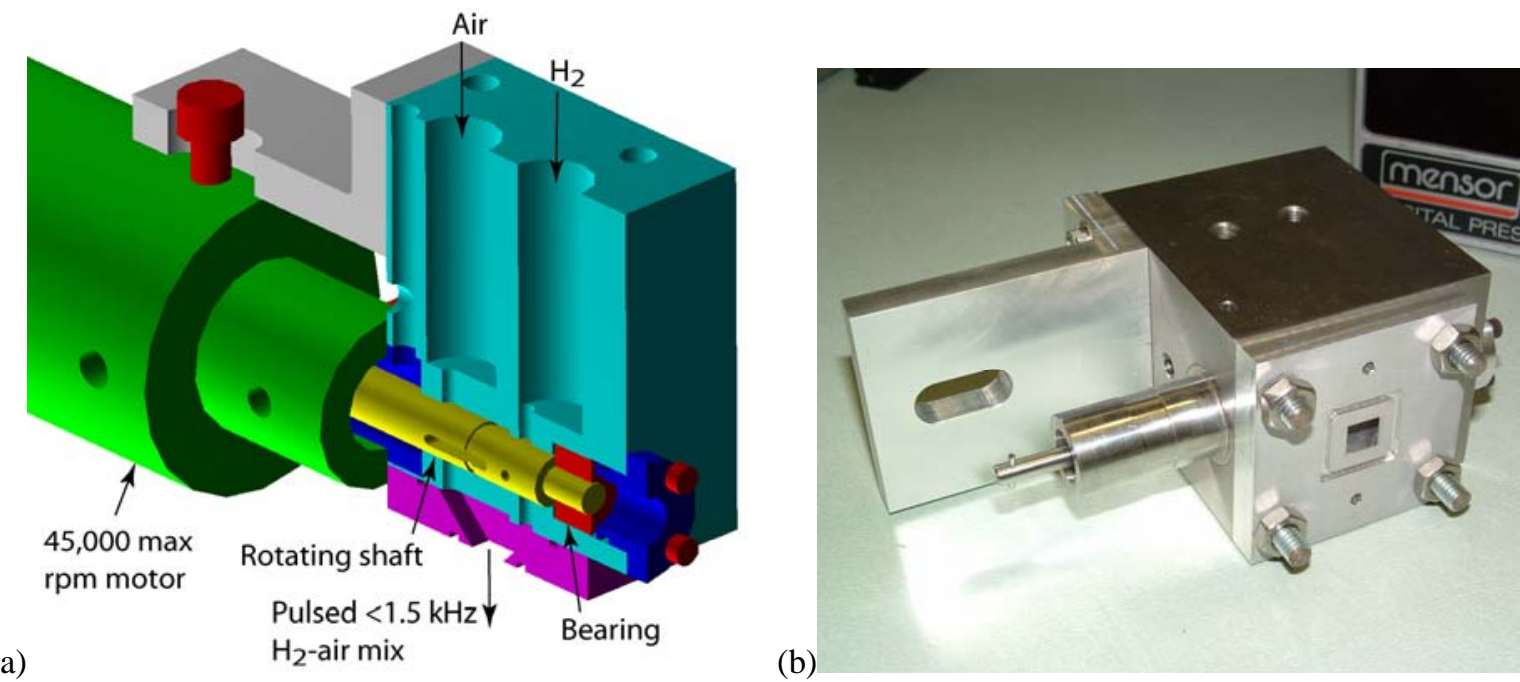

(b)
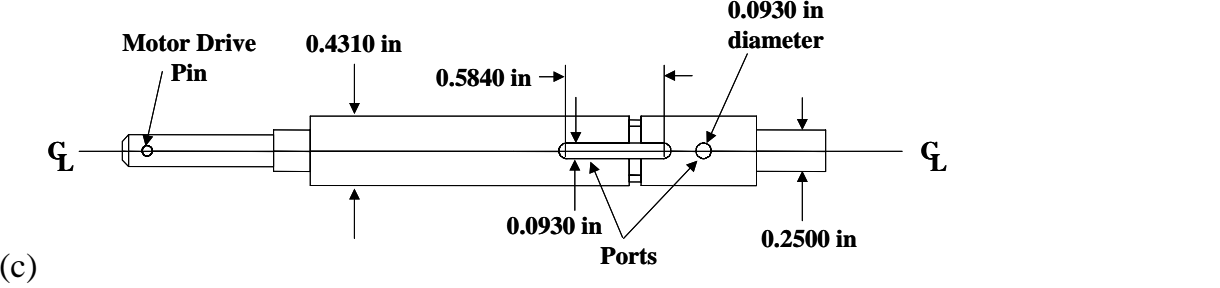

Figure 4. Actuator valve. (a) Partially cut-away view of valve \& motor. (b) Photograph of actuator valve body. (c) Detail of rotating shaft.

Taps were provided in the valve for monitoring both supply $\mathrm{H}_{2}$ and supply air mean pressures. Time-resolved pressure measurements were obtained within the combustion chamber at taps $\mathrm{p} 2$, p3, and p4, located respectively at $x=15.1$ inches, 11.1 inches and 4.0 inches (also shown in Fig. 3(a)). The measurements were by means of 0-250 lbf/in ${ }^{2}$ (psi) PCB-Piezotronics sensors model 113A21 and a 3-channel PCB-Piezotronics signal conditioning unit model 480A21. These gauges only provide AC-coupled (i.e., high frequency pass filtered) pressure. Separate measurements of mean pressure were made using a strain gauge transducer and a pressure tap at $x=15.1$ inches.

High speed pressure data ( 3 channels) and data from the valve shaft position photodiode ( 1 channel) were acquired with a digital oscilloscope, typically at a rate of 50,000 samples per second (per channel). A PC running a Labview program written for the purpose controlled the digital oscilloscope. Data were typically acquired for five seconds and then downloaded to the PC. The PC contained a National Instruments PCI-6025E multipurpose I/O board and preconditioning electronics that allowed simultaneous acquisition of $\mathrm{H}_{2}$ and air flow rate data from the mass flow meters and mean pressure data from the strain gauge pressure transducers. A control voltage could also be sent to the motor controller, increasing or decreasing the motor speed during the course of a run, if desired.

\section{Test Conditions}

During a typical run in which high-frequency pressure data were to be acquired, the valve motor was started, flow rates were established, data acquisition was initiated and the valve motor was ramped either from low to high speed or from high to low speed. The motor speed was held constant, however, during tests in which PLIF or schlieren flow visualization was performed. Typically, high-frequency pressure data were not acquired during such tests.

Six reactant flow rate conditions were studied. The first three (no-combustion cases) had no flow of $\mathrm{H}_{2}$, and three

\begin{tabular}{l|c|c|c} 
& \multicolumn{3}{|c}{ flow rates } \\
\hline & low & middle & high \\
\hline air (lbm/sec) & 0.0178 & 0.0356 & 0.0712 \\
\hline H2 (lbm/sec) & $4.96 \mathrm{E}-04$ & $9.91 \mathrm{E}-04$ & $1.98 \mathrm{E}-03$ \\
\hline air valve (psia) & 45.6 & 87.4 & 161.3 \\
\hline H2 valve (psia) & 46.4 & 79.0 & 140.3 \\
\hline open exit (psia) & 14.8 & 14.9 & 15.3 \\
\hline nozzle exit (psia) & 15.2 & 16.5 & 20.9
\end{tabular}

Table 1. Flow rates, inlet pressures, and tube pressures for the three combustion cases. 
different rates of airflow. The second three had flows of both $\mathrm{H}_{2}$ and air, in stoichiometric proportions, with the air flowing at the rates of the first three cases. Table 1 summarizes the flow rates, the supply inlet pressures, and the tube mean pressure for the three combustion cases. Air flow and air valve pressures are the same for the nocombustion cases, while the tube pressures are very close to ambient (14.7 psia).

Tests were conducted with the nozzle in place at the exit, "nozzle exit", and with the nozzle removed, "open exit”.

\section{E. Optical Setups}

The flowfield near the bottom of the actuator, in the plane of symmetry, was visualized using Planar LaserInduced Fluorescence (PLIF) of the hydroxyl radical, $\mathrm{OH}^{12}$ This radical was probed because it is a good marker of combustion, it can be probed with readily available lasers, and it can provide high signal-to-noise ratio images without requiring gas or particle seeding.

The $\mathrm{OH}$ was excited to fluoresce by ultraviolet light from a frequency doubled, Nd:YAG-pumped dye laser. The Nd:YAG laser was a Spectra Physics DCR-4 laser that produced $~ 550 \mathrm{~mJ} / \mathrm{pulse}$ at $532 \mathrm{~nm}$. To avoid damaging the dye laser, this output was attenuated down to $\sim 250 \mathrm{~mJ} /$ pulse by using Type I frequency doubling and by tuning the Q-switch delay time away from its optimum value. The Nd:YAG pumped a Quantel TDL-51 dye laser operating with Rhodamine-590 laser dye. The dye laser produced about $60 \mathrm{~mJ}$ of light at $566 \mathrm{~nm}$. The frequency and linewidth of this laser were measured using a HighFinesse WS-6 Wavemeter. The linewidth near $566 \mathrm{~nm}$ was measured to be $1.5 \mathrm{~cm}^{-1}$, which is equal to $45 \mathrm{GHz}$. This was somewhat larger than expected and probably resulted in reduced doubling efficiency when this yellow light was converted to $283 \mathrm{~nm}$ using a Spectra Physics Wavelength Extender (WEX). Typically, $5 \mathrm{~mJ}$ of light was obtained at $283 \mathrm{~nm}$. A small fraction of this laser energy was directed to a beam-monitoring station consisting of a small pre-mixed hydrogen-air flame in which $\mathrm{OH}$ laserinduced fluorescence could be monitored by a low-resolution spectrometer equipped with a photomultiplier tube. The laser energy was also monitored using a photodiode. Each day, the laser frequency was scanned and the $\mathrm{OH}$ fluorescence spectrum recorded to confirm that the laser was operating at the desired wavelength. A wavelength of $283.31 \mathrm{~nm}$ was chosen to coincide with the absorption of the Q1(7) transition of the A-X(1,0) band of OH. BenYakar et al. ${ }^{13}$ and others previously used this transition, because the fluorescence intensity of this transition is relatively insensitive to temperature. Thus, with this excitation scheme and for the temperature range expected in this experiment, the $\mathrm{OH}$ PLIF intensity can be qualitatively linked to $\mathrm{OH}$ mole fraction.

A laser light sheet was formed using a $-20 \mathrm{~mm}$ focal length cylindrical lens followed by a $310 \mathrm{~mm}$ focal length spherical lens. These two lenses were separated by approximately $230 \mathrm{~mm}$ so that the spherical lens nearly collimated the beam to about $40 \mathrm{~mm}$ wide in one direction while forming the beam into a « $0.5 \mathrm{~mm}$ thick sheet 310 $\mathrm{mm}$ further downstream. The sheet of laser light was directed up the axis of the combustor via the small slotwindow at the bottom. The sheet focusing position was adjusted to be in the middle of the field of view of the windowed section.

The OH fluorescence was imaged with a Princeton Instruments PI-MAX II intensified CCD camera. Three filters were placed in front of this camera. The first and second filters were a WG-305 and WG-295 used to reject the laser's frequency, which was near $283 \mathrm{~nm}$, while transmitting the $\mathrm{OH}$ fluorescence, which peaks about $315 \mathrm{~nm}$. The third filter, a UG-11, also rejected the laser frequency and transmitted most of the fluorescence but additionally blocked out most of the light in the visible spectrum, attenuating natural flame luminosity as well as room lights. The camera was equipped with a $45 \mathrm{~mm}$ F/1.8 UV transmitting lens, manufactured by EADS Sodern (Cerco Model type 2073). The camera had a 512×512 array, and full frames were acquired without binning. The intensifier was operated at nearly its maximum gain to obtain high signal-to-noise ratio images.

The camera was synchronized with the laser, and both were operated at $10 \mathrm{~Hz}$. A LabView LC880 timing unit that was triggered by an SRS pulse generator controlled the laser and camera timing. The SRS pulse generator was itself triggered by the TTL pulse from the motor shaft of the actuator valve so that the laser and camera systems could be triggered in phase with fuel injection. Typically, a 20-second run consisted of 200 image acquisitions, with each acquisition phased $1 / 200^{\text {th }}$ of an injection cycle past the previous acquisition, while the combustor conditions were kept constant (constant flowrates and constant fuel injection frequency). Thus, each run probed the fuel injection sequence in detail.

A schlieren optical setup was used to provide flow visualization of the actuator exit jet. The setup was a standard Z-Type 2-mirror schlieren system ${ }^{14}$ consisting of a pulsed white light source, concave mirror pair, knife edge (razor blade), and image recording digital CCD camera. Again, the camera was triggered in phase with the fuel injection, with each acquisition phase delayed a small fraction of the injection cycle past the previous acquisition. 


\section{F. Analysis of the Pressure-Time Histories}

Data from the digital oscilloscope were in the form of 4 channels of 250,000 samples each, acquired over 5 seconds: Channel 1 from the valve shaft position photodiode, Channels 2, 3, and 4 from pressure gauges p2, p3, and $\mathrm{p} 4$, respectively. Signals from Channels 2, 3, and 4 were converted from voltage to pressure in $\mathrm{lbf} / \mathrm{in}^{2}$ (psi). For reduction, the data were broken up into 0.1 sec segments of 5000 samples per channel.

For each 0.1 sec segment, the Channel 1 (photodiode) data, which was a string of pulses of finite width, were processed to obtain a string of "delta" functions corresponding to the leading edge of each pulse, occurring once per shaft revolution. The injection frequency was found from the first maximum of the Fast Fourier Transform (FFT) of the processed Channel 1 signal, and multiplying by two to convert shaft revolution frequency to valve opening frequency.

For each 0.1 sec signal segment, the Channels 2, 3, and 4 (pressure) data were analyzed to obtain two separate results. First, the standard deviation (root-mean-square, or RMS, pressure) of the segment was found. The second analysis involved several steps. (i) The segment was band-pass filtered at the injection frequency, using FFT's. (ii) The correlation of the segment with the processed Channel 1 signal segment was found at various time delays. (iii) The first peak of this correlation was found, and consequently the time delay between the photodiode signal and the filtered pressure signal was found. (iv) The phase delay (in cycles) was found by dividing the time delay by the valve opening cycle period. The phase delay needed to be corrected by subtracting a constant, since the signal from the photodiode that collects light from the retroreflective target on the shaft is at arbitrary but fixed phase in relation to the injection. The correction constant (a fixed fraction of a cycle) was found in a separate experiment in which data were acquired when the valve was operated after removing it from the combustor, and one of the pressure transducers was located at the exit of the valve. The corrected phase delay represents the phase delay relative to injection.

Results for RMS pressure and phase delay for each segment of the Channels 2, 3, and 4 data could then be cross-plotted with the valve frequency obtained from the Channel 1 data.

Valve opening (for the air passage) was calculated as a function of phase delay from the geometry of the matching slots in the valve shaft and housing. Using the string of delta functions derived from the Channel 1 data, and the above-described phase delay correction constant, valve opening was inferred for each pressure data sample.

\section{G. Analysis of the Flow Visualization Images}

PLIF image data sets consisted of sequences of 200512 x 512 pixel 16-bit images, each acquired during different injection cycles, at steadily increasing phase delay. Data were analyzed using a specially written MATLAB program. A 3 × 3 Gaussian filter first smoothed the images, the minimum intensity value was subtracted from the whole image, and the image was then normalized by the average intensity of a small region near the top of the field of view where a laser glint was visible. The image was cropped. A boxcar-averaging filter was then applied to the image sequence. The filter used here was 7 images wide, such that each image was averaged with the previous three and subsequent three images in the series. These boxcar-averaged images were stored sequentially as an .avi movie file. In addition, selected images were stored individually. Finally, each boxcar-averaged image was collapsed into a single column by averaging along each row (left and right edges of the image were cropped out, so the average was only taken within the field of view of the detonator window). These columns were stored sequentially in a single image. Such images then show the row-averaged intensity as a function of progress through a single injection cycle (or equivalently, as a function of time during a single injection cycle). The fact that the laser glint region was used to normalize the images is evident in these row-averaged images, as the row corresponding to the laser glint has a constant intensity across one full injection cycle. A selection of these processed images are displayed in Fig. 10 and Fig. 11.

\section{Experimental Results}

\section{A. Pressure Time History Results}

Pressure time history data have been reduced as described to obtain RMS pressure (in psi) and phase delay in cycles, and plotted as a function of injection frequency. Data were acquired at three flow rates, as summarized in Table 1. Results are shown in Figures 5 and 6 for the low flow rate cases, since, in some cases, the resonances were slightly more distinct at the lower flow rates. For each gauge, data are shown from two runs - one in which the injection frequency was ramped up during the run and one in which it was ramped down. Data ramped up are connected by lines; those ramped down are not. Thus, an idea of repeatability may be obtained by comparing data 
to the lines. It may be seen that the repeatability is quite good, except near the resonance peaks where the frequency spacing may be too large to accurately sample the peaks. There is no significant difference between ramping up and down.

Figure 5 shows results derived from pressure time histories for cases where there was only air injected into the actuator and hence, no combustion. Figures (a) and (c) are for runs with the nozzle exit; (b) and (d) are without. The RMS pressure fluctuations, shown in (a) and (b), illustrate that pressure oscillations are excited by the pulsating injection of air into the tube. Many resonances (peaks in the pressure amplitude) may be identified. Many of these are indicated in the figure. The notation $\lambda / 4, \lambda / 2,3 \lambda / 4, \lambda$, etc. refers to the resonant mode. In some cases the notation " $2 \times$ " follows the mode identifier, which indicates that the resonant pressure fluctuation occurs at twice the injection frequency. Otherwise, it may be assumed that the resonance is at the injection frequency.

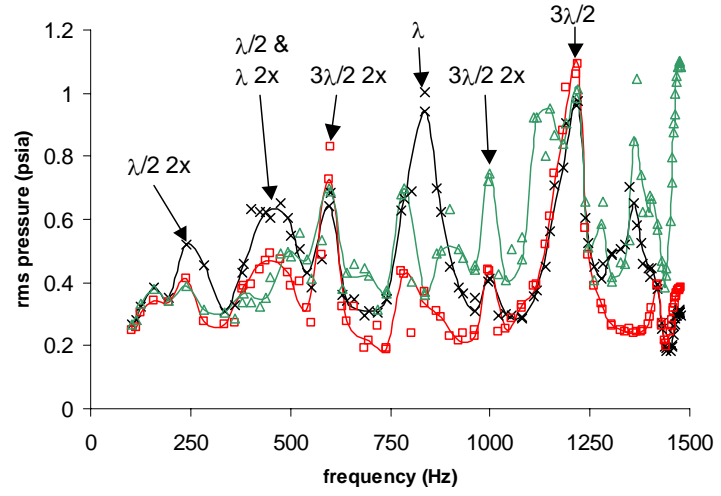

(a)

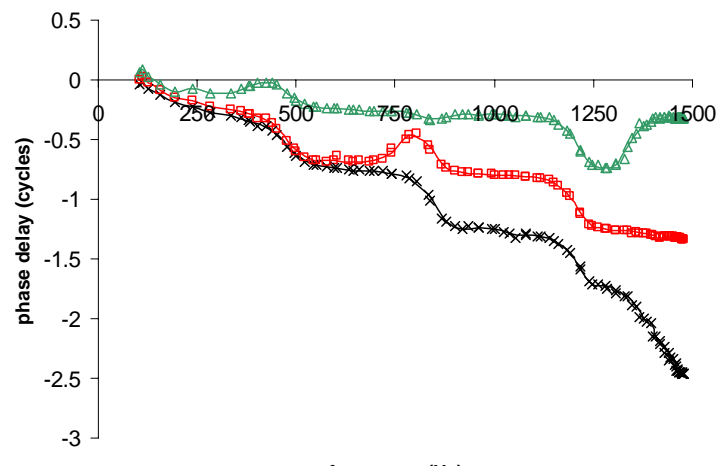

frequency $(\mathrm{Hz})$

(c)

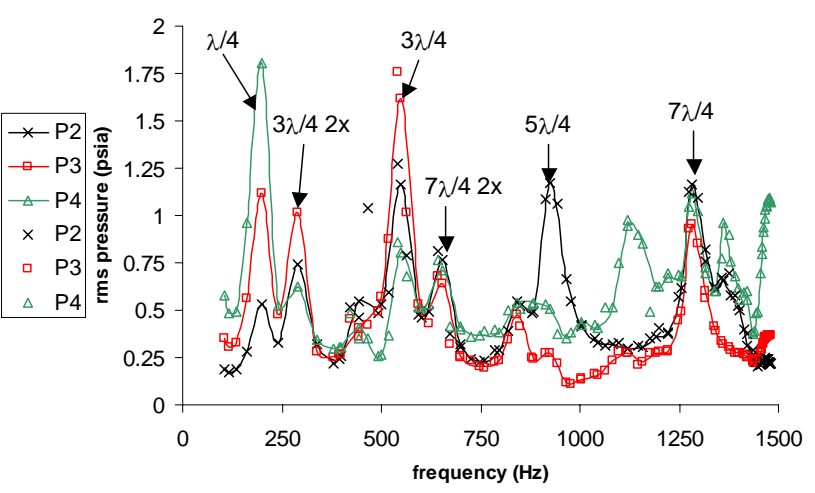

(b)

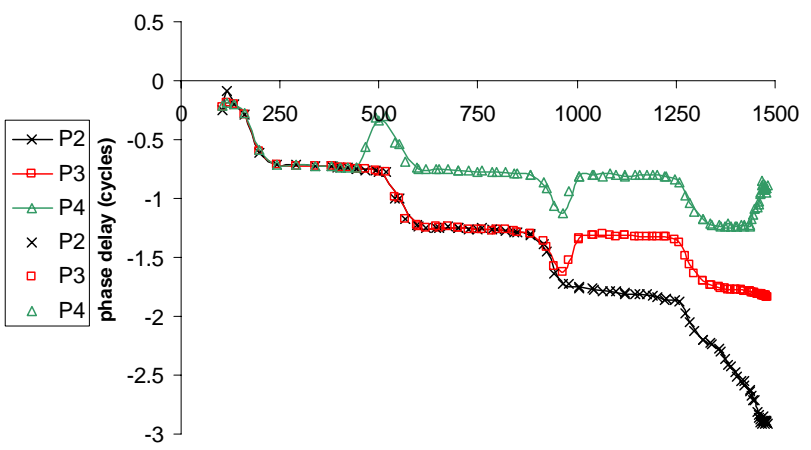

frequency $(\mathrm{Hz})$

(d)

Figure 5. Response of the actuator with air flow at low flow rate as a function of injection frequency: RMS pressures are given in (a), (b), and phase delays in (c), (d). Results with nozzle exit are (a), (c), and with open exit are (b), (d).

Figure 6 shows results derived from pressure time histories for cases with combustion. These are generally similar to the results for air injection, except that a given resonant mode occurs at a higher frequency for combustion, and the RMS pressure fluctuation at the resonance is generally greater. The higher frequencies are to be expected for cases with combustion due to the higher speed of sound of the combustion products.

Identification of the mode in cases where the resonance is at the injection frequency is aided by examination of the phase delay, which is plotted also as a function of injection frequency in Figs. 5 and 6, parts (c) and (d). (Note that the phase delay is only useful in interpreting resonances that occur at the valve frequency, since the pressure signal is bandpass-filtered at that frequency prior to determining phase delay.) To see how the phase delay can be used to help identify the resonance, consider, for example, the $3 \lambda / 2$ resonance at $\sim 1218 \mathrm{~Hz}$ in Fig. 5(a). Recall that a change of sign of pressure amplitude in Figure 2 denotes a phase delay of half a cycle. Thus, the phase of gauge $\mathrm{p} 3$ is delayed half a cycle from $\mathrm{p} 4$, and gauge $\mathrm{p} 2$ is delayed another half cycle from $\mathrm{p} 3$. These expected delays are observed in Fig. 5(c) at the resonance frequency. 


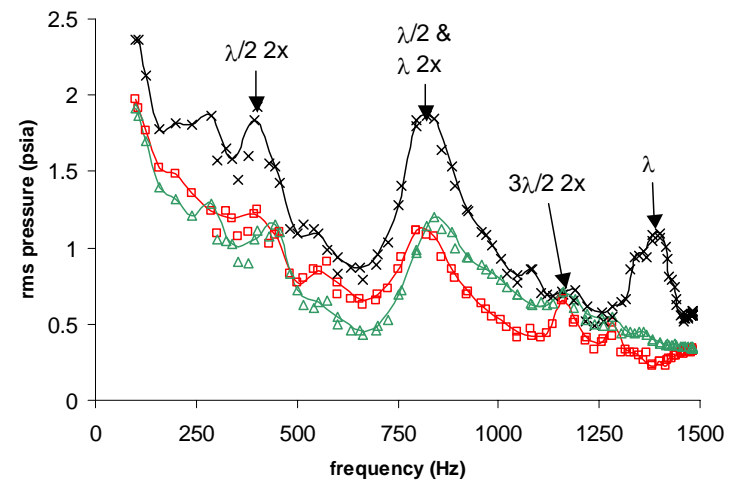

(a)

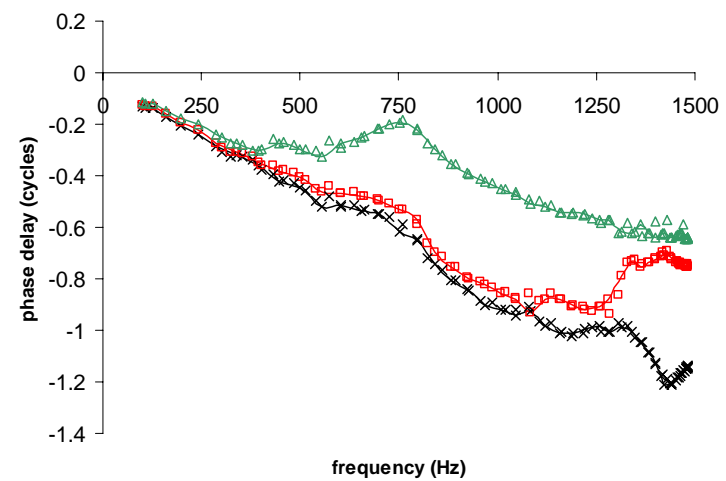

(c)

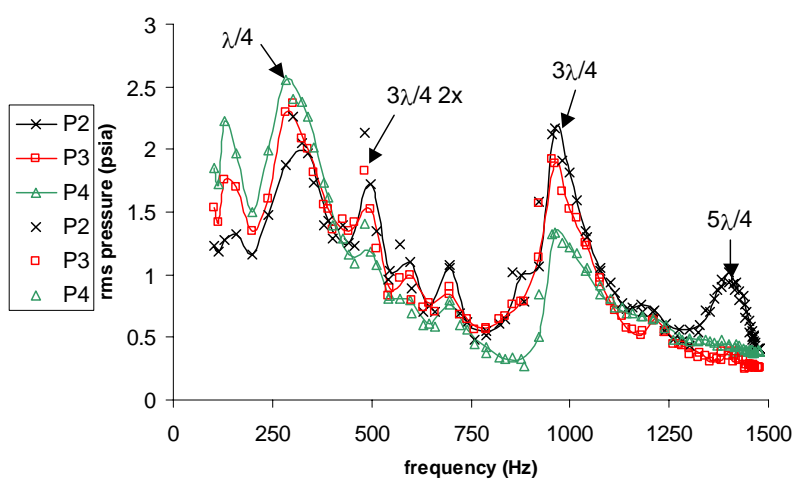

(b)

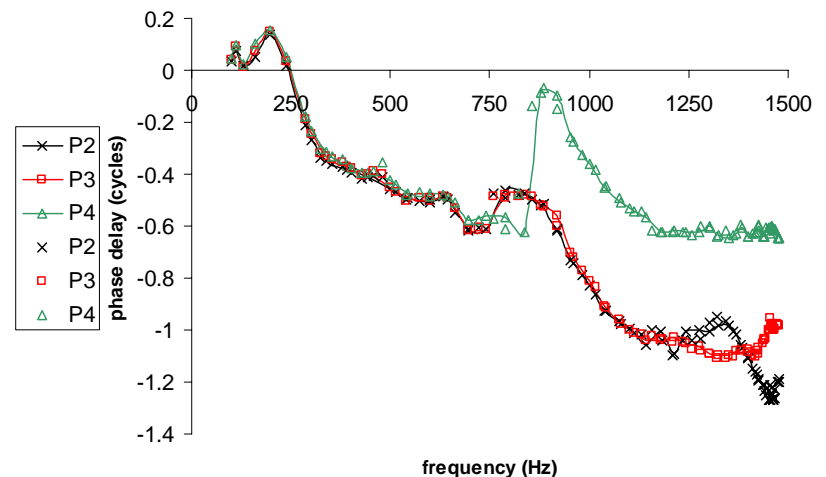

(d)

Figure 6. Response of actuator with combustion at low flow rate as a function of injection frequency. (a), (b), (c), (d) are as for Fig. 6.

Table 2 summarizes the various resonant frequencies and the identified modes. Also shown is the ratio of these resonance frequencies to the ideal resonance frequencies calculated from Equation 1. It may be seen that for air flow (no combustion), the ratio clusters around 1, being between 0.99 and 1.18 for the nozzle exit and between 0.91 and 0.99 for the open exit. This is good agreement, considering the uncertainties in finding the resonant peaks. On the other hand, the ratio values are lower for combustion, being between 0.61 and 0.74 for the nozzle exit and between 0.49 and 0.58 for the open exit. In these calculations, the speed of sound was taken to be the speed of sound in the equilibrium products of adiabatic, isobaric combustion of an initially room temperature stoichiometric mixture of $\mathrm{H}_{2}$ and air. The discrepancy is believed to be due to a lower speed of sound in the tube than that calculated. There are several possible explanations for this. One is that the products may have been cooled by contact with the wall of the combustor. Another is that a significant volume may have been occupied by unreacted reactants. However, it is believed that much of the discrepancy is due to entrainment of cool external air into the actuator. As will be seen below, the difference between mean pressure in the actuator and the surroundings is typically smaller than the pressure fluctuation amplitude, so during portions of the cycle, pressure in the actuator is lower than ambient, and external air will enter the actuator. This is particularly so in the open exit case, consistent with the lower values of the ratio of measured resonant frequencies to ideal for this case.

The changes in peak RMS pressure fluctuation levels at selected resonances as flow rates are increased are summarized in Fig. 7. Both air flow and combustion cases are shown. Figure 7(a) show results for the open exit,

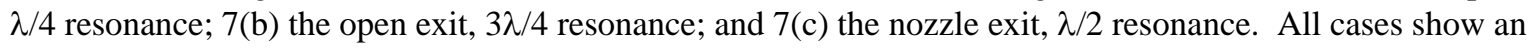
increase in RMS pressure fluctuations with flow rate. The amplitudes with combustion are significantly greater than with air injection alone. However, the amplitudes with combustion at the low flow rates are no more than the amplitudes without combustion at the high flow rates; flow rates between high and low differ by a factor of four. This result is disappointing in that there is an increase in volume by a factor of about 7 for the isobaric combustion of a stoichiometric mixture of $\mathrm{H}_{2}$ and air initially at room temperature. Thus, it might naively be expected that RMS pressure fluctuations with combustion should be similar to those with air (no combustion) at 7 times the volumetric flow rate. A possible explanation is that the reaction occurs too slowly, so that the heat addition associated with mass injection does not occur in phase with it, but is spread across the cycle. 
Figures 8 and 9 show selected time histories of pressure for each gauge, with and without the exit end cap, for cases with high flow rates and combustion. Note that the pressure histories of the different gauges are vertically offset one from another for the sake of clarity. Also shown in these figures is the time dependent variation of the relative opening area of the (air) valve, inferred from the photodiode signal.

Figure 8 shows two cases with the nozzle exit: (a) is a low injection frequency of $183 \mathrm{~Hz}$, and (b) is for injection at $824 \mathrm{~Hz}$, the $\lambda / 2$ resonance. The $183 \mathrm{~Hz}$ case is not at a resonant frequency. In this case, a large pressure spike occurs at each gauge toward the end of the time the valve is open, due to rapid combustion (perhaps a detonation, though not a fully-developed ChapmanJouguet type detonation), and several others of decreasing amplitude follow the first spike of a cycle. For gauge p2, the initial spike is as much as 50 psi above the baseline. The subsequent spikes are associated with wave reflection back and forth in the tube. For the $\lambda / 2$ resonance case, the waveforms are broader, without pronounced multiple oscillations in a cycle, and the pressure rise above the baseline is more than $20 \mathrm{psi}$ for gauge p2. Note the expected half cycle phase difference between gauges $\mathrm{p} 2$ and $\mathrm{p} 4$.

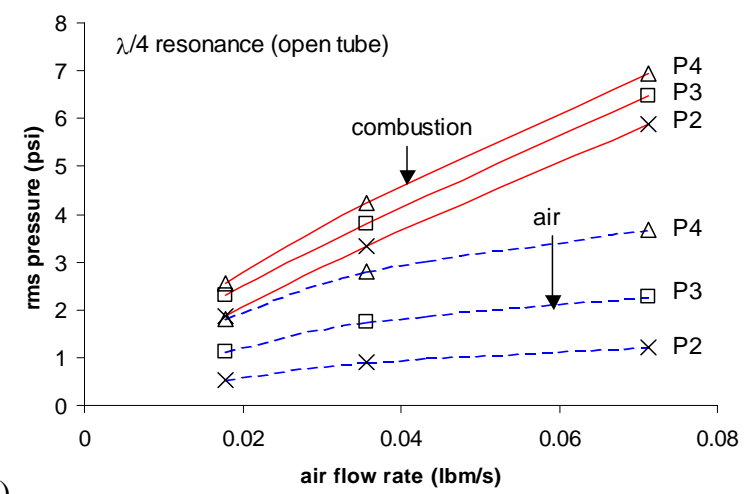

\begin{tabular}{|c|c|c|c|c|}
\hline & \multicolumn{2}{|c|}{ frequency $(\mathrm{Hz})$} & \multicolumn{2}{|c|}{ ratio to theory } \\
\hline mode & comb & air & comb & air \\
\hline$\lambda / 22 x$ & 396 & 238 & 0.695 & 1.183 \\
\hline$\lambda / 2$ & 841 & 440 & 0.738 & 1.094 \\
\hline$\lambda 2 x$ & 841 & 440 & 0.738 & 1.094 \\
\hline $3 \lambda / 22 x$ & 1158 & 596 & 0.677 & 0.988 \\
\hline$\lambda$ & 1399 & 835 & 0.613 & 1.038 \\
\hline $3 \lambda / 2$ & & 1218 & & 1.009 \\
\hline$\lambda / 42 x$ & & & & \\
\hline$\lambda / 4$ & 285 & 199 & 0.500 & 0.989 \\
\hline $3 \lambda / 42 x$ & 498 & 289 & 0.582 & 0.958 \\
\hline $5 \lambda / 42 x$ & & & & \\
\hline $3 \lambda / 4$ & 960 & 550 & 0.561 & 0.912 \\
\hline $7 \lambda / 42 x$ & & 653 & & 0.928 \\
\hline $5 \lambda / 4$ & 1400 & 924 & 0.491 & 0.919 \\
\hline $7 \lambda / 4$ & & 1283 & & 0.911 \\
\hline
\end{tabular}

Table 2. Resonant frequencies at low flow rate for air and combustion cases, and ratios to theory. Resonant frequencies may change slightly with flow rate. Theory assumes speed of sound for air is $1130 \mathrm{ft} / \mathrm{s}$; for combustion products is $3202 \mathrm{ft} / \mathrm{s}$.

(a)
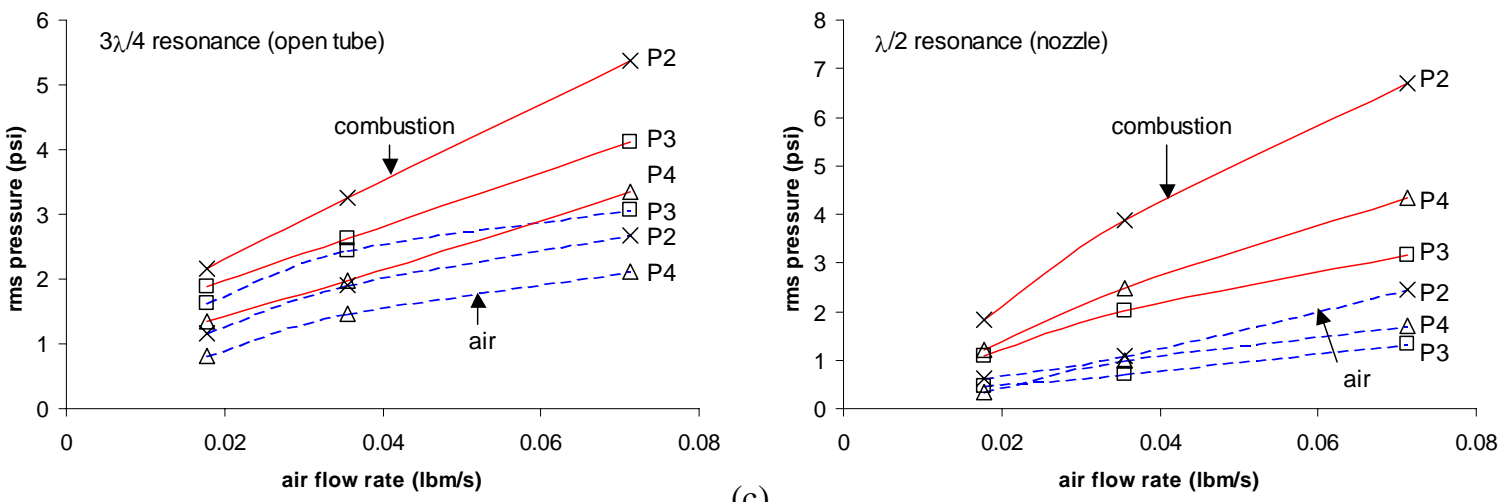

(b)

Figure 7. RMS peak amplitudes of the various resonances (psi) vs. air flow rate for each pressure gauge, air and combustion cases: (a) open exit, $\lambda / 4$ resonance; (b) open exit, $3 \lambda / 4$ resonance; (c) nozzle exit, $\lambda / 2$ resonance.

Figure 9 shows two cases with the open exit: (a) is for injection at $285 \mathrm{~Hz}$, the $\lambda / 4$ resonance, and (b) is for injection at $1078 \mathrm{~Hz}$, the $3 \lambda / 4$ resonance. The $\lambda / 4$ resonance waveform is dominated by a large spike that occurs at the end of injection, perhaps due again to detonation. For p4, this spike is as much as 40 psi above the baseline. Note that all three gauges are in phase, as expected. The $3 \lambda / 4$ resonance waveform is broader, without pronounced multiple oscillations in a cycle, and the pressure rise above the baseline is more than $15 \mathrm{psi}$ for gauge $\mathrm{p} 2$. Note that gauges $\mathrm{p} 3$ and $\mathrm{p} 4$ are in phase, while gauge $\mathrm{p} 2$ is 180 degrees out of phase, as expected. 
In summary, high pressure fluctuations — and in some cases possibly detonations—are observed in this combustion actuator at certain resonant frequencies. Note also that, although data have not been shown here, when the valve frequencies are off-resonance, exceptionally chaotic waveforms are observed, an operating condition that would not be suitable for flow control.

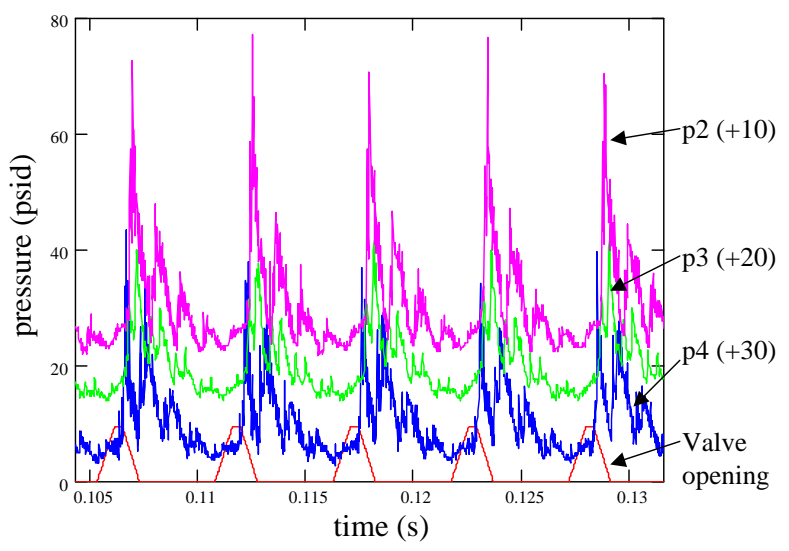

(a)

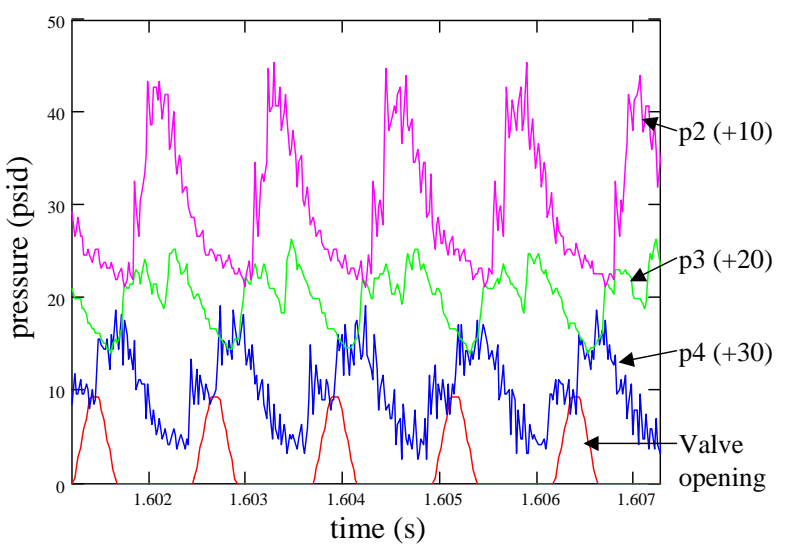

(b)

Figure 8. Selected pressure time histories, high flow rate, combustion, with nozzle exit: (a) excitation at 183 $\mathrm{Hz}$, (b) excitation at $824 \mathrm{~Hz}(\lambda / 2$ resonance).

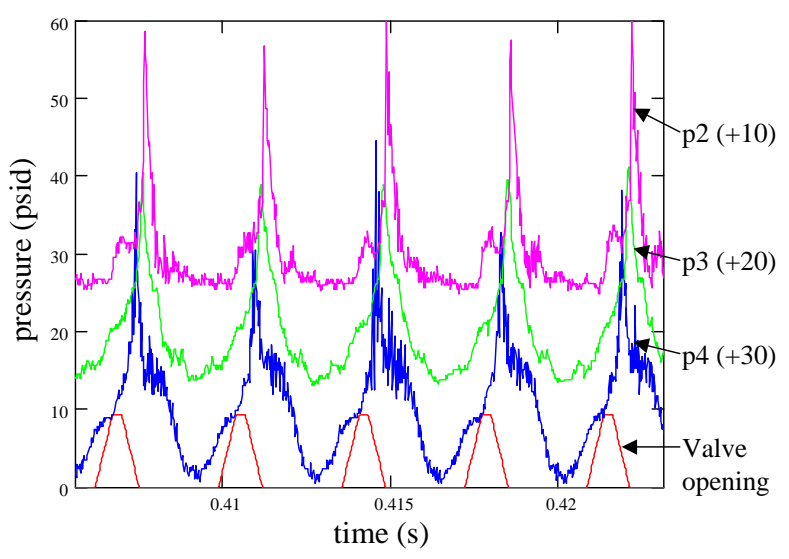

(a)

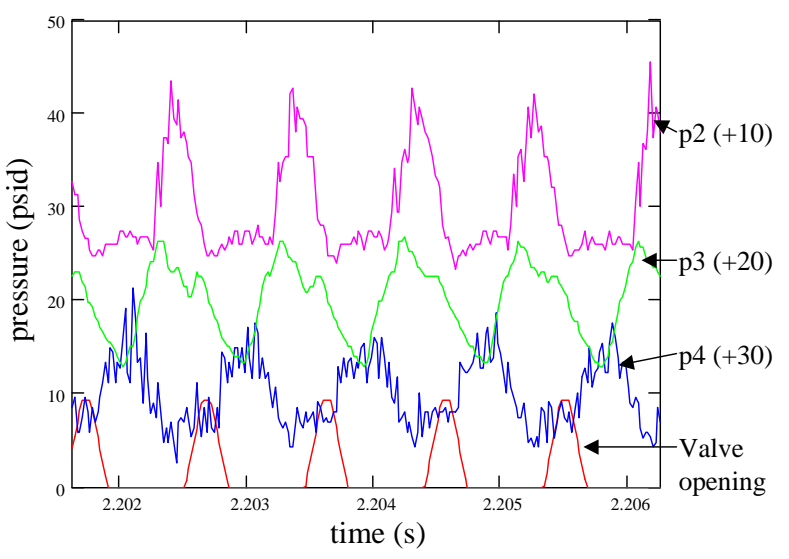

(b)

Figure 9. Selected pressure time histories, high flow rate, combustion, with open exit: (a) excitation at $285 \mathrm{~Hz}$ ( $\lambda / 4$ resonance), (b) excitation at $1078 \mathrm{~Hz}$ ( $3 \lambda / 4$ resonance).

\section{B. Flow Visualization Results}

The results of PLIF visualization of $\mathrm{OH}$ in the symmetry plane of the windowed combustor are presented in Figures 10 and 11. Figure 10 is for two selected runs of the combustor, both at high flow rates. In each part, (a) and (b), a 200-image sequence is summarized. The bottom sequence is 8 selected boxcar-averaged images at equally spaced phase intervals through the cycle. Within these images, the bottom of the image corresponds to the bottom of the combustor, and reactants are injected near the bottom and from the left. The field of view is 1.52 inches wide by 6.0 inches high. The top image shows the row-averaged intensity over the cycle. (Essentially, each boxcaraveraged image is averaged horizontally to form a single-pixel-wide column of the image.) Figure 11 shows the results of eight different runs at different conditions. Several "row-averaged" intensity over the cycle images, like those just described, are matched with plots of pressure at the $\mathrm{p} 4$ gauge, which is within the field of view of these PLIF images (towards the top). Also shown is the relative opening of the valve, indicating the entry of reactants into the combustor. The plots have been carefully matched to the images so that at a given horizontal position, the phase angles in each are the same. In these images, the color map ranges from black (no $\mathrm{OH}$ emission) through blue and orange, to white (indicating the highest levels of $\mathrm{OH}$ ). The exact correspondence of the color map with $\mathrm{OH}$ emission level may vary from one run to the next, but should be consistent within a run. For the purposes of 
interpretation of these images, we will assume that regions of high $\mathrm{OH}$ emission (and high $\mathrm{OH}$ levels) are also regions in which recent heat release has taken place due to chemical reaction. We will assume that dark regions are regions of unburned reactants or regions that have been cooled by expansion.

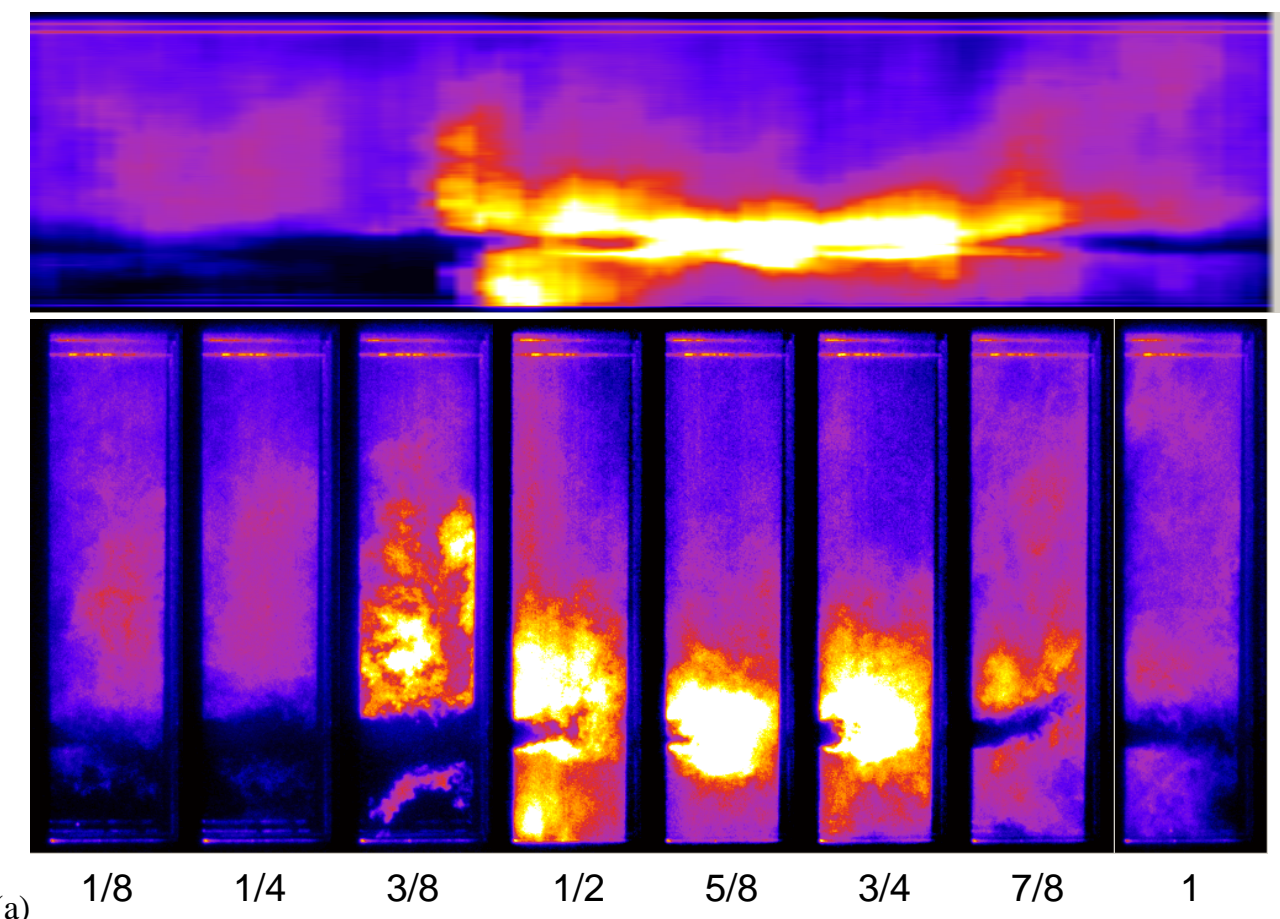

(a)
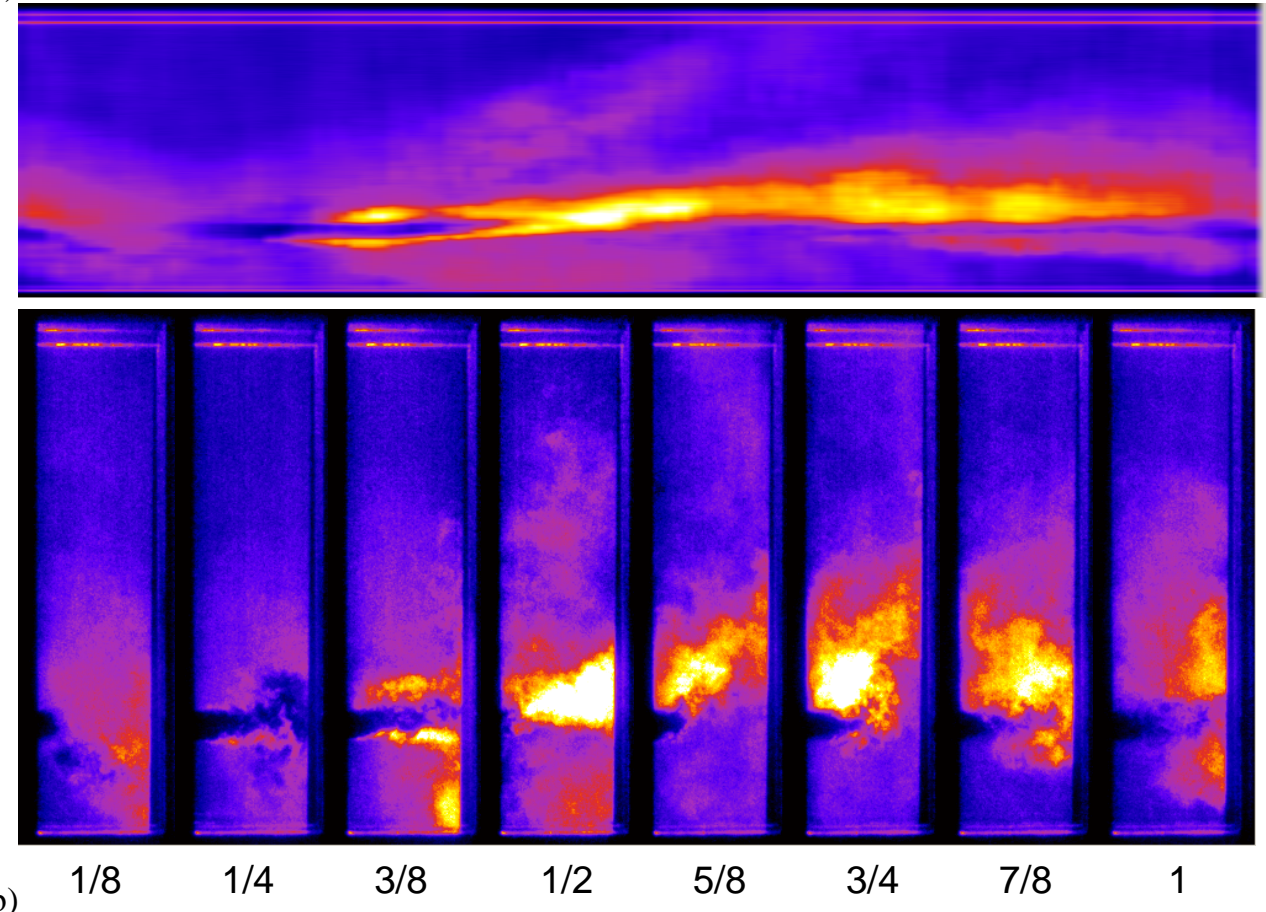

Figure 10. PLIF images of $\mathrm{OH}$ fluorescence. (a) and (b) each contain an image showing row-averaged intensity as a function of progress through a single injection cycle (top) and sequence of 8 images at equally spaced points in the cycle (bottom). Two cases with high flow rates: (a) open exit, $285 \mathrm{~Hz}$ ( $\lambda / 4$ resonance); (b) nozzle exit, $824 \mathrm{~Hz}$ ( $\lambda / 2$ resonance). 

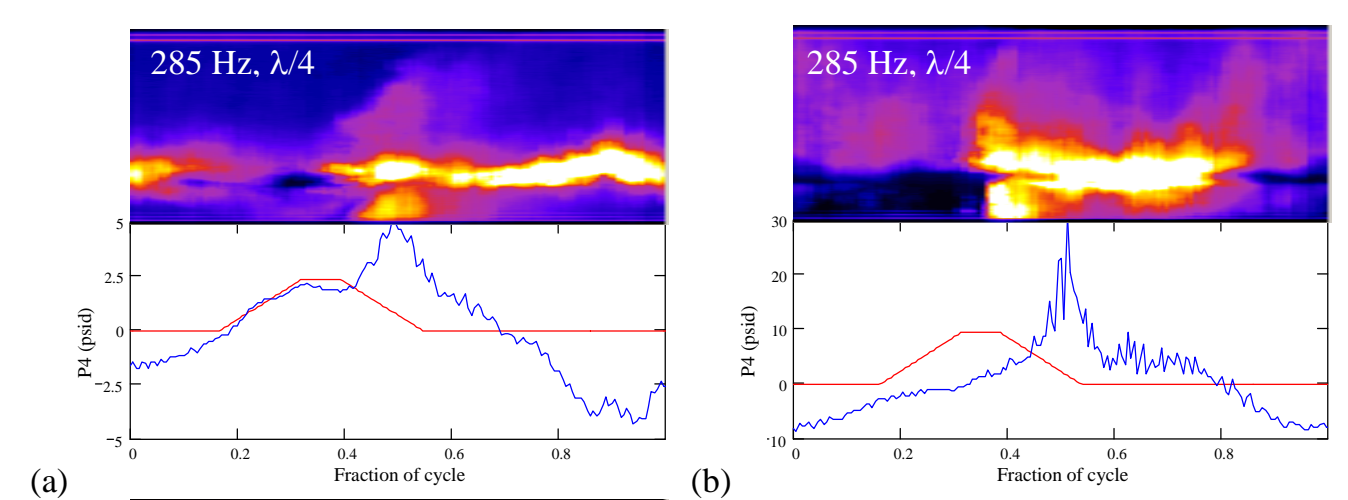

(a)

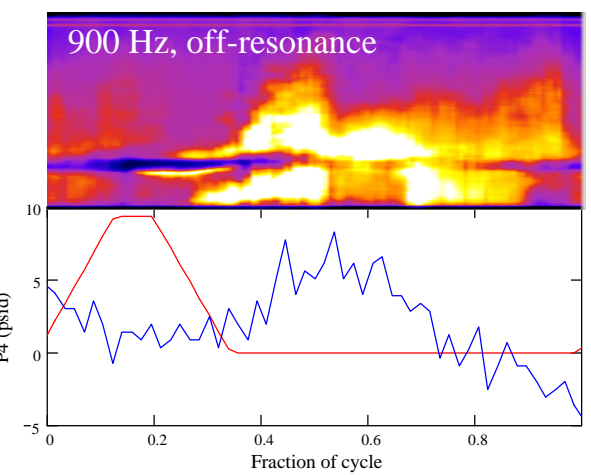

(b)

(c)

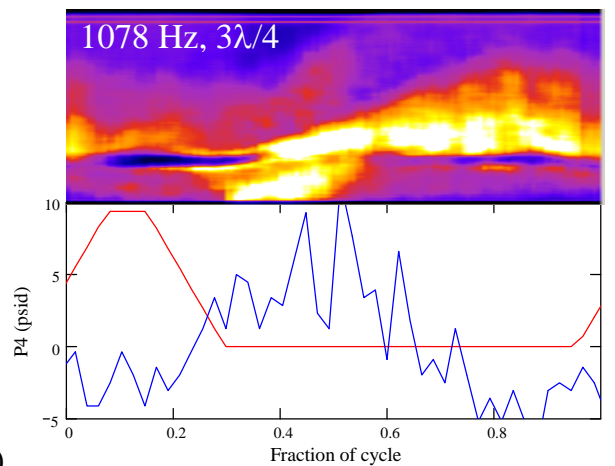

(d)

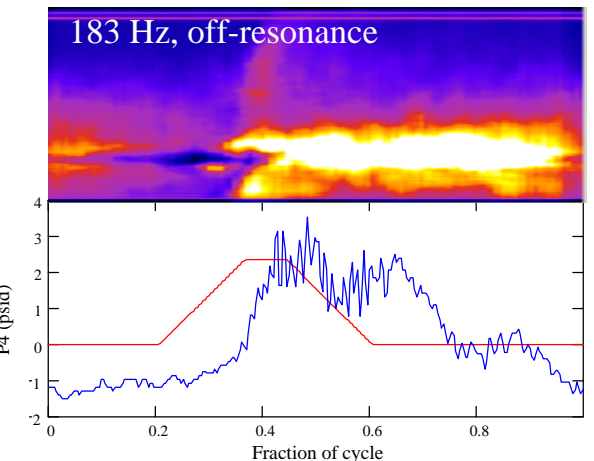

(e)
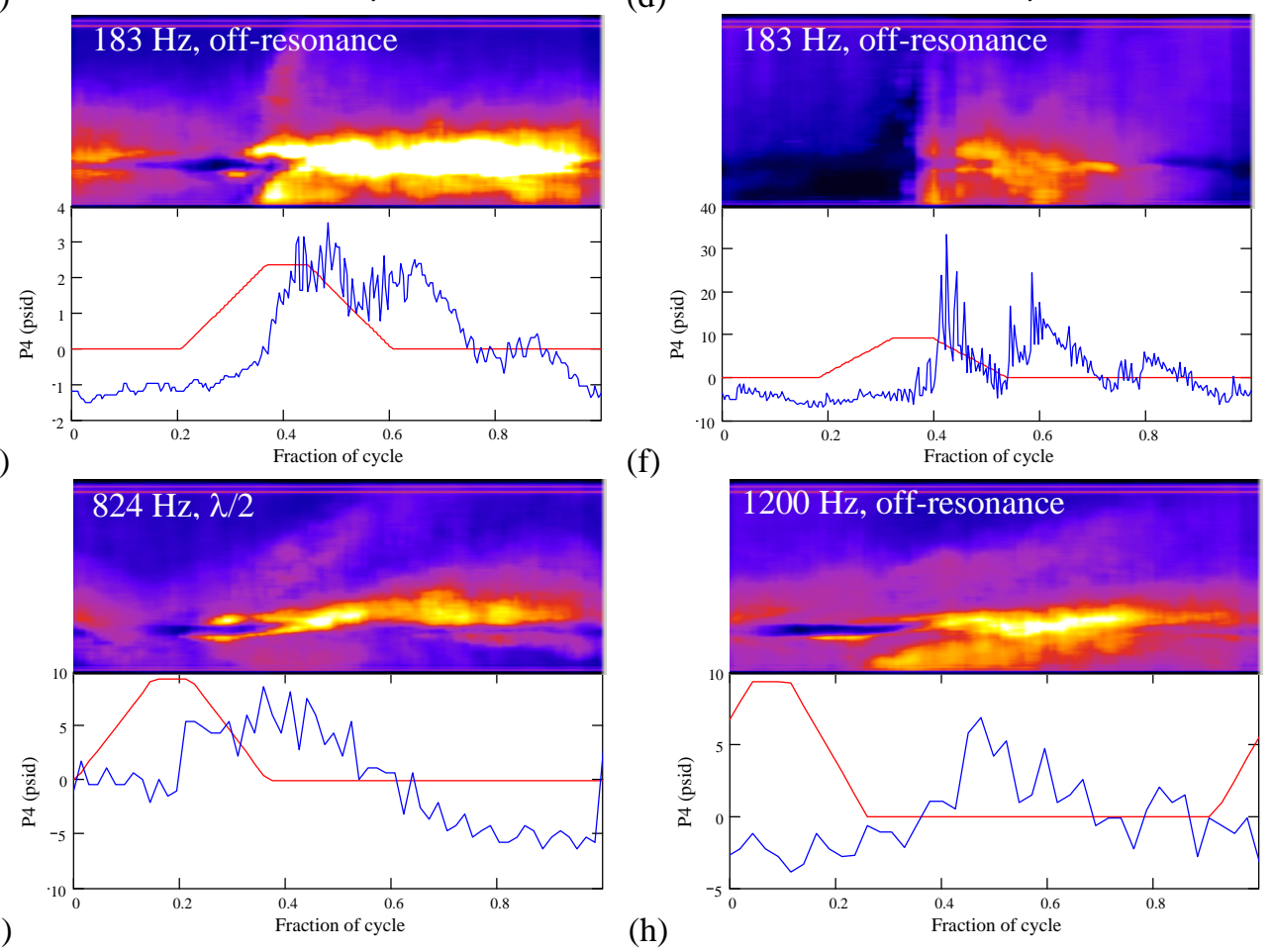

(f)

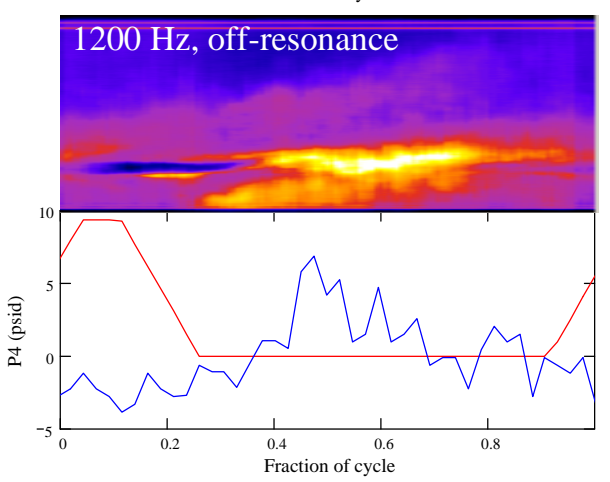

(h)

Figure 11. Each figure has a PLIF image showing row-averaged intensity (top), and a plot showing pressure for gauge $\mathrm{p} 4$ in blue and valve relative opening in red (bottom). Horizontal axis is fraction of cycle (image and plot). Eight cases are shown: (a) and (e) are for low flow and the rest for high flow; (a)-(d) are for open exit, (e)-(h) are for nozzle exit. Frequencies vary.

Figure 10(a) is for the open exit with a valve frequency of $285 \mathrm{~Hz}$, exciting the $\lambda / 4$ resonance. It may be seen that little combustion occurs for the first $3 / 8$ of the cycle. A dark region at the bottom of each image indicates a region of cool gas (lacking $\mathrm{OH}$ ) or injected reactants. Between $3 / 8$ and $1 / 2$ cycle, reaction occurs abruptly, as 
evidenced by regions with high levels of $\mathrm{OH}$. Between $3 / 4$ and 7/8 cycle, the high levels of $\mathrm{OH}$ end. Comparison with pressure, in Fig. 11(b), reveals that the appearance of high $\mathrm{OH}$ levels near mid-cycle coincides with the tail end of the time the valve is open and with the previously discussed large pressure spike. The disappearance of high $\mathrm{OH}$ levels at around 0.8 of a cycle corresponds to a pressure reduction to minimum levels, presumably due to the expansion wave that reflects from the open exit and propagates back into the tube. Clearly, combustion is strongly coupled to wave motions in this case.

Figure 10(b) is for the nozzle exit with the valve opening at $824 \mathrm{~Hz}$, exciting the $\lambda / 2$ resonance. High levels of $\mathrm{OH}$ again appear around $3 / 8$ and $1 / 2$ cycle, but these persist through $7 / 8$ cycle. The region of high $\mathrm{OH}$ levels is more localized (vertically) due to the smaller volume of reactants injected during the cycle than in 10(a). (Note that the reactant flow rate is the same in each case, but the cycle period, and hence the quantity of fuel injected in the cycle, is shorter by a factor of 285/824.) Comparison with pressure, in Fig. 11(g), again reveals that the appearance of high $\mathrm{OH}$ levels coincides with the tail end of the time the valve is open and with the previously discussed pressure rise. However, the reduction in $\mathrm{OH}$ emission is not as pronounced during the latter half of the cycle, when pressure levels are low. It is suspected that heat release due to combustion is extended over the portion of the cycle when pressure is low. Heat addition during this phase of the cycle is undesirable since it will tend to suppress rather than enhance pressure fluctuations. However, one should be cautious about drawing too strong a conclusion regarding this matter. High levels of $\mathrm{OH}$ may not correlate directly with heat release, but rather $\mathrm{OH}$ at near equilibrium levels will convect with the products of reaction, after the heat release has ended.

The effect of the quantity of fuel injected in one cycle for otherwise identical cases may be seen in Fig. 11 by comparing (a) and (b), at $285 \mathrm{~Hz}$ ( $\lambda / 4$ resonance) with open exit, or (e) and (f), at $183 \mathrm{~Hz}$ with nozzle exit. Figs (a) and (e) are at low flow while (b) and (f) are at high. In both cases, it may be seen that combustion persists longer

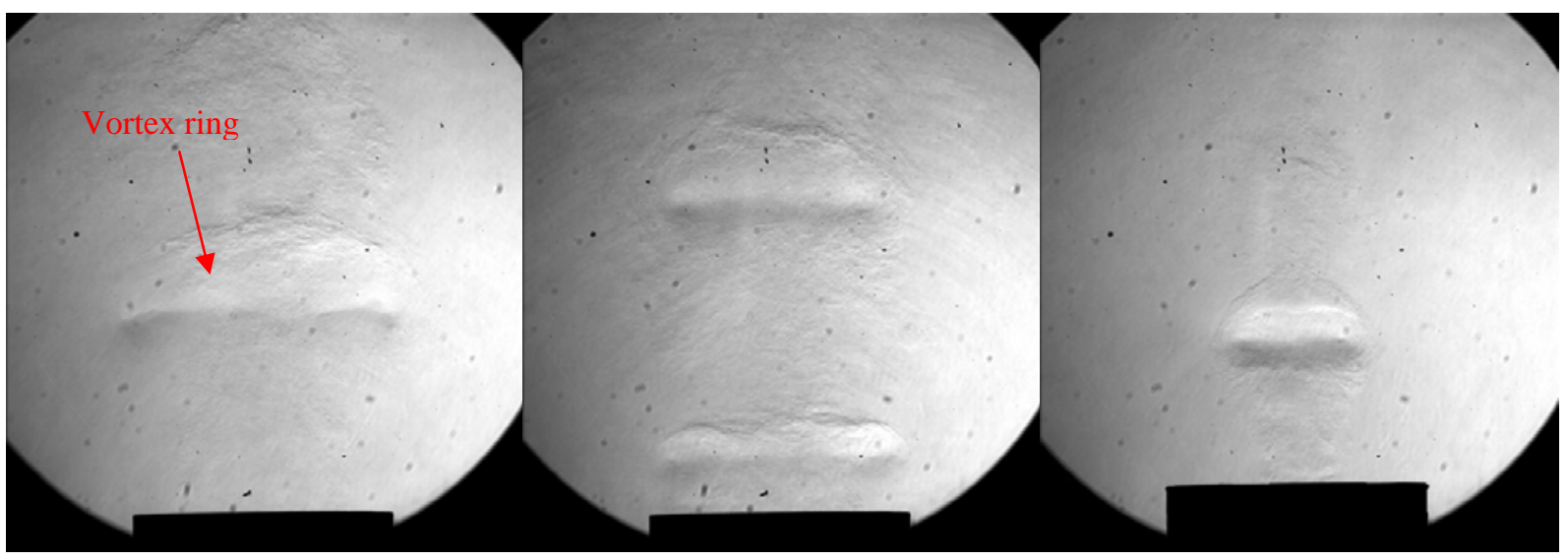

Figure 12. Schlieren images of jet above combustor exit with air only at high flow rate (a) $\lambda / 4$ (open exit,

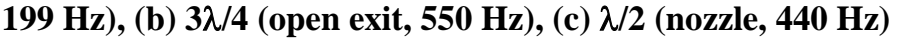

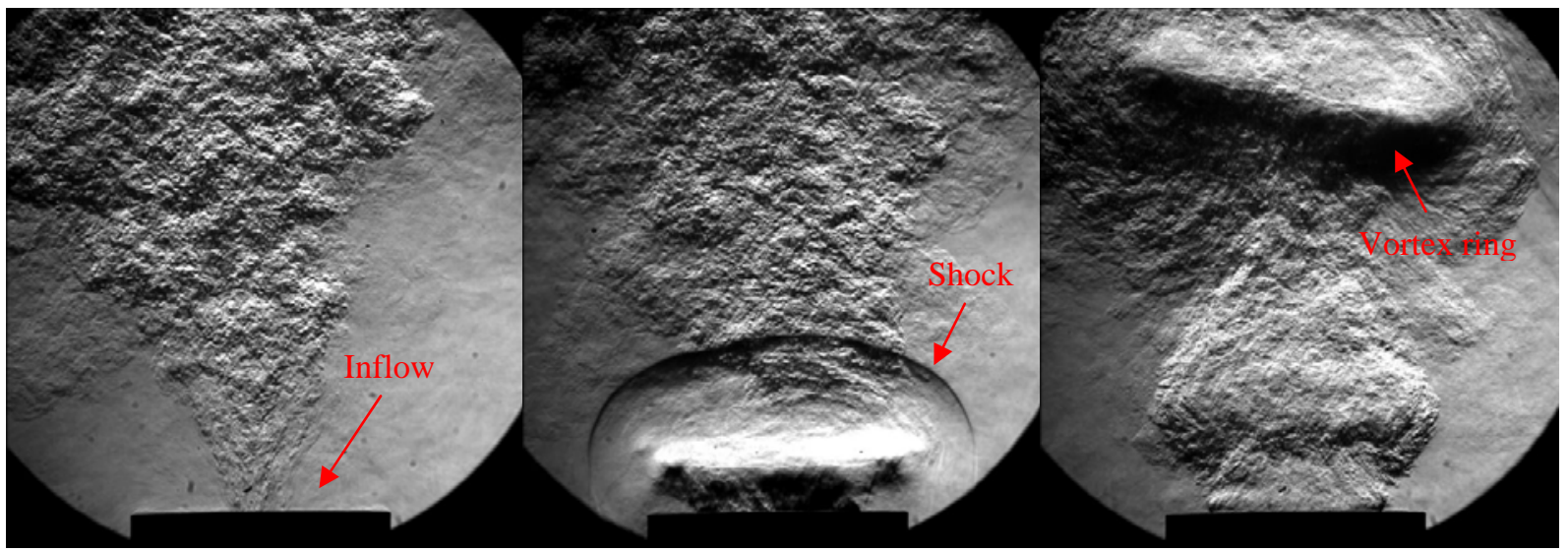

Figure 13 Schlieren images of jet above combustor exit with combustion at high flow rate, $\lambda / 4$ (open exit, 285 $\mathrm{Hz}$ ), at various phases of the cycle, (a) start, (b) $0.19^{\text {th }}$ of cycle, (c) $0.36^{\text {th }}$ of cycle. 
through the cycle for low flow than for high. Other resonant cases are shown in Figs. 11(d), $1078 \mathrm{~Hz}, 3 \lambda / 4$ resonance, and (g), $824 \mathrm{~Hz}, \lambda / 2$ resonance. The $\mathrm{OH}$ and pressure results for these two cases are quite similar. Note the vertical rise and slight fall of the region of high $\mathrm{OH}$, and the absence of high $\mathrm{OH}$ at the bottom, between 0.5 and 1 cycle. This is due to expansion of the gas, in the latter part of the cycle, which both cools the gas at the bottom, reducing $\mathrm{OH}$ levels, and convects the injected reactants upwards. Compare with results for two off resonance cases, shown in Fig. 11(c) $900 \mathrm{~Hz}$ open exit, and (h) 1200, Hz nozzle exit. In both cases, this vertical rise and fall is absent, and high $\mathrm{OH}$ levels persist near the bottom.

In summary therefore, variations in $\mathrm{OH}$ level may be correlated with injection of reactants into the combustion chamber, and with local pressure. Higher reactant charge in the combustion chamber is associated with reaction (high OH levels) that extends along a greater length of the tube, but for a shorter period of time. Resonant behavior is associated with a vertical rise and fall in the region of high $\mathrm{OH}$ levels after injection, and the suppression of $\mathrm{OH}$ due to gas expansion near the bottom surface.

\section{Visualization of Pulsating Air Jet}

Schlieren images of the pulsating jet discharging from the actuator are shown in Figs. 12, 13, and 14. A selected image from each of three resonant cases with air flow at the high flow rate is shown in Fig. 12: (a) and (b) are for the open exit at 199 $\mathrm{Hz}$ and $550 \mathrm{~Hz}$ respectively, and (c) is for the nozzle exit at 440 Hz. Axially symmetric vortex rings are observed as a consequence of the density gradients they induce. In sequences of images not shown, these vortex rings are seen to convect upwards at constant velocity. The velocity of these rings was estimated from these images, and the results are listed in Table 3. The velocity is much greater for the nozzle exit case, (c), perhaps because the mean velocity of the flow at the nozzle exit is greater.

Figure 13 shows a sequence of three images at various phases of a cycle with combustion at high flow rate, open exit, $285 \mathrm{~Hz}$. In (a), a large plume of combustion products that tapers to a narrow waist at the open exit of the actuator may be seen. This tapering occurs because, at this point in the cycle, the pressure in the actuator is low, and air from both the plume and the ambient is being drawn into the actuator. In (b) a slug of combustion products is exiting the actuator at high speed (see Table 3 ), creating a shock wave that is seen in other images to propagate outward rapidly. In (c), the shock wave has exited the field of view and an axially symmetric vortex has propagated to nearly the top of the field. Figure 14 is a single image with combustion at high flow rate, open exit, $1078 \mathrm{~Hz}$. In this case, the vortex rings are less clearly visible among the turbulent eddies of hot and colder air that surrounds the jet above the nozzle. The speed of the vortex rings is also less (Table 3), so that two rings are visible in this image: one just above the nozzle exit and one about $70 \%$ of the distance up the image.

\section{Conclusions}

A prototype actuator was constructed and tested using pulsed combustion or detonations within a resonant tube to provide a pulsed jet flow suitable for flow control in high-speed applications. This actuator utilized a high-speed valve, capable of $100 \mathrm{~Hz}$ to $1500 \mathrm{~Hz}$ injection cycle rates, to supply a mixture of $\mathrm{H}_{2}$ and air to the actuator combustion chamber. The actuator was instrumented with high-speed pressure transducers, and equipped with windows. Planar Laser Induced Fluorescence (PLIF) was employed to visualize the flow in the actuator while schlieren was used to visualize the pulsed jet above the exit. A number of resonant operating conditions were observed, with both an open exit and a nozzle exit condition. Pressure fluctuations were the greatest, most ordered, and most periodic, in addition to the combustion (as imaged by PLIF) being most strongly coupled to the wave action, at these resonant conditions. The highest pressure-fluctuation levels, along with periodic detonation-like behavior, were found when the charge of reactants (more strictly, the fraction of the volume of the tube filled by 
reactants) in any one cycle was largest. Due to flow rate limitations of our valve, this occurred at relatively low frequencies $(285 \mathrm{~Hz})$. It is speculated that, by reducing the cross-sectional area of our actuator while maintaining its length and existing reactant flow rates, periodic controlled detonations can be extended to the higher frequency resonant modes. Reducing the length of the tube will increase the frequencies of specific resonant modes, allowing detonations to occur at higher frequencies for the lowest order resonance. Work is currently in progress to test these hypotheses, and also to test the capabilities of this actuator in controlling separation in a transonic nozzle.

\section{Acknowledgments}

The first author would like to thank the NASA Langley Research Center for its support of this research through a Research Cooperative Agreement, NCC1-03011, Technical Monitor Dr. A.H. Auslender. We would also like to thank Stephen B. Jones for setting up the schlieren system used in this research, and other valuable assistance.

\section{References}

${ }^{1}$ Funk, R., Parekh, D., Crittenden, T. and Glezer, A., “Transient Separation Control using Pulse Combustion Actuation,” $1^{\text {st }}$ Flow Control conference, 24-26 June 2002, Paper\# AIAA 2002-3166.

2 NASA Langley Research Center, Fact Sheet\# FS-2000-06-52-LaRC.

${ }^{3}$ McCormick, B. W., Aerodynamics, Aeronautics and Flight Mechanics, $2^{\text {nd }}$ Edition, Wiley, pp. 86-109, 1995.

${ }^{4}$ Grossman, K. R., Cybyk, B. Z., and VanWie, D. M., "Sparkjet Actuators for Flow Control," $41^{\text {st }}$ AIAA Aerospace Sciences Meeting \& Exhibit, 6-9 Jan. 2003, Paper\# AIAA-2003-0057.

${ }^{5}$ Crittenden, T. and Glezer, A., Funk, R., Parekh, D., “Combustion-Driven Jet Actuators for Flow Control,” $31^{\text {st }}$

AIAA Fluid Dynamics Conference \& Exhibit, 11-14 June 2001, Paper\# AIAA 2001-2768.

${ }^{6}$ Rinehart, C., McMichael, J. M., and Glezer, A., "Transitory Flow and force Development on a Body of Revolution Using Synthetic Jet Actuation,” Paper\# AIAA 2003-0618.

${ }^{7}$ Honohan, A.M., Amitay, M., Glezer, A., “Aerodynamic Control using Synthetic Jets,” AIAA Fluids 2000

Conference \& Exhibit, 19-22 June 2003, Paper\# AIAA-2000-2401.

${ }^{8}$ Kuo, K. K., Principles of Combustion, Wiley, 1986, pp. 231-283.

${ }^{9}$ B. T. Beck, A. D. Cutler, J. P. Drummond, S. B. Jones, “A Resonant Pulse Detonation Actuator for High-Speed Boundary Layer Separation Control,” 11th International Symposium On Flow Visualization, August 9-12, 2004, University of Notre Dame, Notre Dame, Indiana, USA, August 9-12, 2004.

${ }^{10}$ Diedrich, G. (translated by Kahane, A.), “The Aero-Resonator Power Plant of the V-1 Flying Bomb,” Project Squid TM Pr-4, Princeton University, 30 June 1948.

${ }^{11}$ Kailasanath, K., "Review of Propulsion Applications of Detonation Waves,” AIAA Journal, Vol. 38, No. 9, Sept 2000.

${ }^{12}$ Eckbreth, A. C., Laser Diagnostics For Combustion Temperature And Species, Gordon and Breach Pub., 1996.

${ }^{13}$ Ben-Yakar, A., Kamel, M. R., Morris, C. I., and Hanson, R.K., "Hypersonic Combustion and Mixing Studies using Simultaneous OH-PLIF and Schlieren Imaging”, AIAA Paper 98-0940.

${ }^{14}$ Settles, G. S., Schlieren and Shadowgraph Techniques, Springer, pp. 42-44, 1949. 